\title{
How Well Does the Finite Fourier Transform Approximate the Fourier Transform?
}

\author{
CHARLES L. EPSTEIN \\ University of Pennsylvania \\ Department of Mathematics and \\ Laboratory for Structural NMR Imaging \\ In memory of Hannah Peretsman Breen
}

\begin{abstract}
We show that the answer to the question in the title is "very well indeed." In particular, we prove that, throughout the maximum possible range, the finite Fourier coefficients provide a good approximation to the Fourier coefficients of a piecewise continuous function. For a continuous periodic function, the size of the error is estimated in terms of the modulus of continuity of the function. The estimates improve commensurately as the functions become smoother. We also show that the partial sums of the finite Fourier transform provide essentially as good an approximation to the function and its derivatives as the partial sums of the ordinary Fourier series. Along the way we establish analogues of the Riemann-Lebesgue lemma and the localization principle. (C) 2004 Wiley Periodicals, Inc.
\end{abstract}

\section{Introduction}

Let $\mathcal{S}_{N}$ denote the set of complex sequences of length $N+1$,

$$
\mathcal{S}_{N}=\left\{\left\langle a_{0}, a_{1}, \ldots, a_{N}\right\rangle: a_{j} \in \mathbb{C}, j=0, \ldots, N\right\} .
$$

For each $N \in \mathbb{N}$, the finite Fourier transform (FFT) is the map from $\mathcal{S}_{N}$ to itself defined by

$$
\mathcal{F}_{N}\left(\left\langle a_{j}\right\rangle\right)_{k}=\frac{1}{N+1} \sum_{j=0}^{N} a_{j} e^{-\frac{2 \pi i j k}{N+1}} .
$$

Using formula (1.2), this sequence can be extended to all $k \in \mathbb{Z}$ and is periodic of period $N+1$. For our purposes, it is more natural to think of $k$ as going from

$$
k_{\min }=-\left\lfloor\frac{N-1}{2}\right\rfloor \text { to } k_{\max }=\left\lfloor\frac{N}{2}\right\rfloor .
$$


The inverse of $\mathcal{F}_{N}$ is given by

$$
\mathcal{F}_{N}^{-1}\left(\left\langle a_{j}\right\rangle\right)=\sum_{k=k_{\min }}^{k_{\max }} a_{j} e^{\frac{2 \pi i j k}{N+1}} .
$$

A principal application of the FFT is to approximately compute samples of the Fourier transform of a function. If $f$ is a function defined on $[0,1]$, then we define

$$
\tilde{f}_{N, k}=\frac{1}{N+1} \sum_{j=1}^{N} f\left(\frac{j}{N+1}\right) e^{-\frac{2 \pi i j k}{N+1}} .
$$

It is apparent that the sum on the right-hand side of equation (1.4) is a Riemann sum for the integral defining the $k^{\text {th }}$ Fourier coefficient

$$
\hat{f}(k)=\int_{0}^{1} f(x) e^{-2 \pi i k x} d x .
$$

In the signal processing literature, it is often asserted that

$$
\hat{f}(k) \approx \tilde{f}_{N, k},
$$

at least for $|k| \ll N$, though there is evident trepidation in asserting this for values of $k$ comparable to $\frac{N}{2}$. This is because the integrand becomes highly oscillatory and therefore the standard estimates indicate that, for $|k|$ near to $\frac{N}{2}$, this Riemann sum may not provide an accurate approximation to the integral.

In this note we show that this concern is entirely unfounded and that, in fact, $\tilde{f}_{N, k}$ provides a uniformly good estimate for $\hat{f}(k)$ for $k \in\left[k_{\min }, k_{\max }\right]$. We consider functions that are either continuous or piecewise continuous with finitely many points of discontinuity (as 1-periodic functions) on $[0,1]$. This is a natural restriction in a context where one is sampling. To simplify the notation, we restrict our attention to $N=2 M+1$, so that

$$
k_{\min }=-M \quad \text { and } \quad k_{\max }=M .
$$

Similar results are true for $N=2 M$. The asymptotic properties of the Fourier coefficients, as $|k|$ tends to infinity, are due to cancellations that occur in the integral in (1.5). Our results show that essentially the same cancellations occur in the finite sum in (1.4). In examples we show that, if the samples are not uniformly spaced, then these cancellations may fail to occur.

If, as a 1-periodic function, $f$ has continuous derivatives, then the approximation in equation (1.6) is commensurately better. Moreover, we show that the FFT partial sum

$$
\tilde{f}_{N}(x)=\sum_{k=k_{\min }}^{k_{\max }} \tilde{f}_{N, k} e^{2 \pi i k x}
$$


is almost as good an approximation to $f$ as the usual partial sum

$$
f_{N}(x)=\sum_{k=k_{\min }}^{k_{\max }} \hat{f}(k) e^{2 \pi i k x} .
$$

We also show that the one-dimensional FFT has the same localization properties as the Fourier transform.

Some of the results in this paper are part of the folklore in the world of numerical analysis. One should consult the work of Henrici [4] or of Gottlieb and Shu [3] and the references contained therein. In most of the published work, it is assumed that the functions under consideration are piecewise analytic, whereas no such assumption is needed for our methods to apply. I am not aware of results as sharp or proofs that are as simple and direct as those provided here. The focus in the work of Gottlieb et al. is on how to use the exact knowledge of a finite number of Fourier coefficients to obtain a very accurate approximation to a piecewise analytic function, even when it has jump discontinuities. In [8] methods are described for approximating Fourier coefficients outside of the range $\left[k_{\min }, k_{\max }\right]$. These techniques involve using higher-order interpolants to approximate the function from the sampled data.

In [1] Auslander and Grünbaum consider the problem of estimating the error in using the discrete Fourier transform to compute the Fourier transform of a squareintegrable function. Because an $L^{2}$-function does not have well-defined values at points, this analysis also includes a model for sampling both the function and its Fourier transform. Using the Cauchy-Schwarz inequality, the authors derive relative bounds for the errors in the Fourier coefficients that depend on the sampling models and the frequency but are independent of the function.

\section{Some Background Material}

Our analysis is based on classical results in approximation theory related to Jackson's theorem. A good reference for this material is [7]. The facts we use from Fourier theory can be found in $[2,5]$.

To state the result, we need to define the modulus of continuity of a function. Let $f$ be a function defined on a domain $D$; then the modulus of continuity of $f$ is defined by

$$
\omega_{f}(\delta)=\sup _{\substack{x_{1}, x_{2} \in D \\\left|x_{1}-x_{2}\right| \leq \delta}}\left|f\left(x_{1}\right)-f\left(x_{2}\right)\right| .
$$

An exponential polynomial of order $M$ is a function of the form

$$
p(x)=\sum_{k=-M}^{M} \alpha_{k} e^{2 \pi i k x}, \quad \alpha_{k} \in \mathbb{C} .
$$


We denote the set of such functions by $\mathcal{T}_{M}$. If $f$ is a continuous, 1-periodic function defined on $[0,1]$, then, for each $M \in \mathbb{N}$, there is a function $p^{*} \in \mathcal{T}_{M}$ that is a best uniform approximation to $f$; that is,

$$
\left\|f-p^{*}\right\|_{\infty}=\inf _{p \in \mathcal{T}_{M}}\|f-p\|_{\infty} .
$$

Theorem 1.3 in [7] reads as follows:

THEOREM 2.1 [7, theorem 1.3] If $f(x)$ is a continuous 1-periodic function on $[0,1]$, then the best uniform approximation $p^{*}$ to $f$ from $\mathcal{T}_{M}$ satisfies

$$
\left\|f-p^{*}\right\|_{\infty} \leq 6 \omega_{f}\left(\frac{1}{2 \pi M}\right) .
$$

Arguing as in the case of Jackson's theorem, we easily derive the following corollary:

COROLLARY 2.2 If $f$ is a continuous 1-periodic function on $[0,1]$ with l continuous 1-periodic derivatives, then the best uniform approximation $p^{*}$ to $f$ from $\mathcal{T}_{M}$ satisfies

$$
\left\|f-p^{*}\right\|_{\infty} \leq 6^{l+1} \frac{\omega_{f}^{l}\left(\frac{1}{2 \pi M}\right)}{(2 \pi M)^{l}} .
$$

Here and in what follows, $\omega_{f}^{l}$ is the modulus of continuity of $f^{[l]}$.

For completeness we include the case that $f$ is analytic:

COROLLARY 2.3 If $f$ is a continuous 1-periodic function on $[0,1]$ with a bounded analytic extension to a strip of the form

$$
\mathcal{S}_{a}=\{z:|\mathfrak{\Im} z|<a\}
$$

then the best uniform approximation $p^{*}$ to $f$ from $\mathcal{T}_{M}$ satisfies

$$
\left\|f-p^{*}\right\|_{\infty} \leq \frac{2 K e^{-2 \pi a M}}{e^{2 \pi a}-1} .
$$

Here $K=\sup \left\{|f(z)|: z \in \mathcal{S}_{a}\right\}=\|f\|_{L^{\infty}\left(\mathcal{S}_{a}\right)}$.

Sharp results for the Fourier transform are often proven using these results. The following lemma indicates how they can be applied to study the finite Fourier transform:

LEMMA 2.4 If $p \in \mathcal{T}_{M}$, then

$$
\tilde{p}_{2 M, k}=\hat{p}(k) \quad \text { for }-M \leq k \leq M .
$$

The proof is an elementary computation. The relation in equation (2.7) does not hold for $|k|>M$. For $|k|>M$ the Fourier coefficient $\hat{p}(k)$ vanishes, whereas $\left\langle\tilde{p}_{2 M, k}\right\rangle$ is a $(2 M+1)$-periodic sequence. 


\section{The Continuous Case}

In this section we state and prove our results for continuous 1-periodic functions. The case of piecewise continuous functions is treated in the next section.

THEOREM 3.1 For $M \in \mathbb{N}$ and $f$, a continuous 1-periodic function defined on $[0,1]$, we have the estimates

$$
\left|\tilde{f}_{2 M, k}-\hat{f}(k)\right| \leq 2\left\|p^{*}-f\right\|_{\infty} \quad \text { for }|k| \leq M,
$$

where $p^{*} \in \mathcal{T}_{M}$ is defined by equation (2.3).

PROOF: Since $p^{*} \in \mathcal{T}_{M}$, a simple calculation shows that, for $|k| \leq M$, we have

$$
\begin{aligned}
& \left|\tilde{f}_{2 M, k}-\tilde{p}_{2 M, k}^{*}\right| \\
& \quad=\left|\frac{1}{2 M+1} \sum_{j=0}^{2 M}\left[f\left(\frac{j}{2 M+1}\right)-p^{*}\left(\frac{j}{2 M+1}\right)\right] e^{-\frac{2 \pi i j k}{2 M+1}}\right| \\
& \quad \leq\left\|f-p^{*}\right\|_{\infty} .
\end{aligned}
$$

In the second line we use the triangle inequality. On the other hand, Lemma 2.4 implies that

$$
\begin{aligned}
\left|\hat{f}(k)-\tilde{p}_{2 M, k}^{*}\right| & =\left|\hat{f}(k)-\widehat{p}^{*}(k)\right| \\
& =\left|\int_{0}^{1}\left[f(x)-p^{*}(x)\right] e^{-2 \pi i k x} d x\right| \\
& \leq\left\|f-p^{*}\right\|_{\infty},
\end{aligned}
$$

where again we use the triangle inequality to go from the second to the third line. Combining equations (3.2) and (3.3) with one further application of the triangle inequality gives the result.

As a corollary of this theorem and Corollaries 2.2 and 2.3, we have the following:

COROLlary 3.2 Suppose that $f$ is a continuous, 1-periodic function with $l \geq 0$ continuous 1-periodic derivatives; then

$$
\left|\tilde{f}_{2 M, k}-\hat{f}(k)\right| \leq 12 \frac{6^{l} \omega_{f}^{l}\left(\frac{1}{2 \pi M}\right)}{(2 \pi M)^{l}},
$$

for $|k| \leq M$. If $f$ has a bounded analytic extension to $\mathcal{S}_{a}$, then, for $|k| \leq M$,

$$
\left|\tilde{f}_{2 M, k}-\hat{f}(k)\right| \leq \frac{4 K e^{-2 \pi a M}}{e^{2 \pi a}-1}, \quad \text { where } K=\|f\|_{L^{\infty}\left(\mathcal{S}_{a}\right)} .
$$

If $f$ satisfies the hypotheses of Corollary 3.2 then, for $m \leq l$, we can express the Fourier coefficients of $f^{[m]}$ in terms of those of $f$ :

$$
\widehat{f^{[m]}}(k)=(2 \pi i k)^{m} \hat{f}(k) \text {. }
$$


Using these relations in the estimate, (3.4) gives

$$
\left|(2 \pi i k)^{m} \tilde{f}_{2 M, k}-\widehat{f^{[m]}}(k)\right| \leq 12 \frac{6^{l} \omega_{f}^{l}\left(\frac{1}{2 \pi M}\right)}{(2 \pi M)^{l-m}} \quad \text { for }|k| \leq M, m \leq l .
$$

Thus, for $|k| \leq M,(2 \pi i k)^{m} \tilde{f}_{2 M, k}$ is a good approximation to $\widehat{f^{[m]}}(k)$.

So far we have only considered the absolute errors entailed in replacing $\{\hat{f}(k)\}$ by $\left\{\tilde{f}_{2 M, k}\right\}$. We now turn briefly to a consideration of the relative errors. Using the fact that

$$
\int_{0}^{1} f(x) e^{-2 \pi i k x} d x=\sum_{j=0}^{k-1} \int_{\frac{j}{k}}^{\frac{j+1}{k}}\left[f(x)-f\left(\frac{j}{k}\right)\right] e^{-2 \pi i k x} d x,
$$

we easily establish that, if $f$ has $l$ periodic derivatives, then

$$
|\hat{f}(k)| \leq \frac{\omega_{f}^{l}\left(\frac{1}{k}\right)}{(2 \pi|k|)^{l}} \quad \text { if } k \neq 0 .
$$

Comparing this to the estimate in (2.5), we see that the sequences $\left\{\tilde{f}_{2 M, k}\right\}$ have the correct rate of decay. Using the right-hand side of equation (3.9) as a proxy for the rate of decay of $\{\hat{f}(k)\}$, we estimate the relative error:

$$
\frac{\left|\tilde{f}_{2 M, k}-\hat{f}(k)\right|}{|\hat{f}(k)|} \approx \frac{12 \omega_{f}^{l}\left(\frac{1}{2 \pi M}\right)}{\omega_{f}^{l}\left(\frac{1}{|k|}\right)}\left(\frac{6|k|}{M}\right)^{l} .
$$

While the relative error remains bounded for $|k| \leq M$, it is only small for indices that satisfy $6|k| \ll M$.

A corollary of Theorems 2.1 and 3.1 is a uniform Riemann-Lebesgue lemma for finite Fourier coefficients:

COROLlary 3.3 Suppose that $f$ is a continuous 1-periodic function; then

$$
\left|\tilde{f}_{2 M, k}\right| \leq|\hat{f}(k)|+6 \omega_{f}\left(\frac{1}{2 \pi M}\right)
$$

which implies that there is a decreasing sequence $\left\langle a_{k}\right\rangle$, tending to 0 , so that, for every $M$, we have

$$
\left|\tilde{f}_{2 M, k}\right| \leq a_{k} \quad \text { for }|k| \leq M
$$

We now turn to the comparison between $\tilde{f}_{2 M}(x)$ and $f_{2 M}(x)$.

THEOREM 3.4 There is a universal constant $C$ so that, if $f$ is a continuous 1periodic function, then

$$
\left|\tilde{f}_{2 M}(x)-f_{2 M}(x)\right| \leq C \log M\left\|f-p^{*}\right\|_{\infty},
$$

where $p^{*} \in \mathcal{T}_{M}$ is a best uniform approximation. 
PROOF: For this argument we use the Dirichlet kernel

$$
D_{M}(x)=\frac{\sin [\pi(2 M+1) x]}{\sin (\pi x)} .
$$

It is well-known that

$$
f_{2 M}(x)=\int_{0}^{1} D_{M}(x-y) f(y) d y .
$$

A simple calculation shows that $\tilde{f}_{2 M}$ is given by a Riemann sum for this integral:

$$
\tilde{f}_{2 M}(x)=\frac{1}{2 M+1} \sum_{j=0}^{2 M} D_{M}\left(x-\frac{j}{2 M+1}\right) f\left(\frac{j}{2 M+1}\right) .
$$

To prove the theorem, we observe that

$$
\begin{aligned}
&\left|\tilde{f}_{2 M}(x)-f_{2 M}(x)\right| \\
& \leq\left|\tilde{f}_{2 M}(x)-p^{*}(x)\right|+\left|p^{*}(x)-f_{2 M}(x)\right| \\
& \leq \frac{1}{2 M+1} \\
& \quad \cdot \sum_{j=0}^{2 M}\left|D_{M}\left(x-\frac{j}{2 M+1}\right)\left[f\left(\frac{j}{2 M+1}\right)-p^{*}\left(\frac{j}{2 M+1}\right)\right]\right| \\
& \quad+\int_{0}^{1}\left|D_{M}(x-y)\left[p^{*}(y)-f(y)\right]\right| d y \\
& \leq\left\|f-p^{*}\right\|_{\infty} \\
& \cdot\left[\frac{1}{2 M+1} \sum_{j=0}^{2 M}\left|D_{M}\left(x-\frac{j}{2 M+1}\right)\right|+\int_{0}^{1}\left|D_{M}(x-y)\right| d y\right] .
\end{aligned}
$$

It is a classical result that both the sum and the integral in the last line are bounded by a constant times $\log M$; see [7].

As a corollary of the proof, we have the following criterion for $\left\langle\tilde{f}_{2 M}\right\rangle$ to converge uniformly to $f$ :

COROLlaRY 3.5 If $f$ is a continuous, 1-periodic function whose modulus of continuity satisfies

$$
\omega_{f}(\delta)=o\left(|\log \delta|^{-1}\right),
$$

then the FFT partial sums $\left\langle\tilde{f}_{2 M}\right\rangle$ converge uniformly to $f$ on $[0,1]$. If $f$ has $l$ continuous periodic derivatives and $\omega_{f}^{l}$ satisfies the estimate in (3.17), then, for each $1 \leq j \leq l$, the sequence $\left\langle\partial_{x}^{j} \tilde{f}_{2 M}\right\rangle$ converges uniformly to $\partial_{x}^{j} f$. 
ProOF: Let $p^{*} \in \mathcal{T}_{M}$ be a best uniform approximation to $f$. We use the triangle inequality to conclude that

$$
\left|\tilde{f}_{2 M}(x)-f(x)\right| \leq\left|\tilde{f}_{2 M}(x)-p^{*}(x)\right|+\left|p^{*}(x)-f(x)\right| .
$$

Arguing as above and applying Theorem 2.1, we see that

$$
\left|\tilde{f}_{2 M}(x)-f(x)\right| \leq(C \log M+6) \omega_{f}\left(\frac{1}{2 \pi M}\right) .
$$

The estimate in equation (3.17) implies that the right-hand side of equation (3.19) tends to zero as $M$ tends to infinity. If $f$ has $l$ derivatives, then the same argument applies to $\left\langle\partial_{x}^{j} \tilde{f}_{2 M}\right\rangle$ for $1 \leq j \leq l$.

The last two results show that, among trigonometric polynomials, the approximation to $f$ (and its derivatives) afforded by $\left\langle\tilde{f}_{2 M}\right\rangle$ is very close to optimal.

\section{Piecewise Continuous Functions}

If $f$ is a piecewise continuous function with jump discontinuities at $\left\{x_{0}, \ldots\right.$, $x_{p}$, then we can express $f$ as a sum $f^{\mathrm{c}}+f^{\mathrm{j}}$ where $f^{\mathrm{c}}$ is a continuous 1-periodic function and $f^{\mathrm{j}}$ is a function of the form

$$
f^{\mathrm{j}}(x)=\sum_{j=1}^{p}\left(\alpha_{j} x+\beta_{j}\right) \chi_{\left[x_{p-1}, x_{p}\right)}(x) .
$$

The continuous part can be treated by using results from the previous section, leaving only the jump terms. Since the sum in (4.1) is finite, for the purposes of obtaining estimates it suffices to consider a single term

$$
g=(\alpha x+\beta) \chi_{\left[x_{0}, x_{1}\right]}(x) .
$$

Both $\hat{g}(k)$ and $\tilde{g}_{2 M, k}$ can be computed explicitly:

$$
\begin{aligned}
& \hat{g}(k)=\alpha {\left[\frac{x_{0} e^{-2 \pi i x_{0}}-x_{1} e^{-2 \pi i x_{1}}}{2 \pi i k}-\frac{e^{-2 \pi i x_{0}}-e^{-2 \pi i x_{1}}}{4 \pi^{2} k^{2}}\right] } \\
&+\beta\left[\frac{e^{-2 \pi i x_{0}}-e^{-2 \pi i x_{1}}}{2 \pi i k}\right], \\
& \tilde{g}_{2 M, k}=\alpha\left[h\left(\frac{2 \pi k}{2 M+1}\right) \frac{\frac{j_{0}}{2 M+1} e^{-\frac{2 \pi i j_{0} k}{2 M+1}}-\frac{j_{1}}{2 M+1} e^{-\frac{2 \pi i\left(j_{1}+1\right) k}{2 M+1}}}{2 \pi i k}\right. \\
&\left.-h\left(\frac{2 \pi k}{2 M+1}\right)^{2} \frac{e^{-\frac{2 \pi i\left(j_{0}+1\right) k}{2 M+1}}-e^{-\frac{2 \pi i\left(j_{1}+1\right) k}{2 M+1}}}{4 \pi^{2} k^{2}}\right] \\
&+\beta\left[h\left(\frac{2 \pi k}{2 M+1}\right) \frac{e^{-\frac{2 \pi i j_{0} k}{2 M+1}}-e^{-\frac{2 \pi i\left(j_{1}+1\right) k}{2 M+1}}}{2 \pi i k}\right] .
\end{aligned}
$$


Here $h$ is defined by

$$
h(x)=\frac{i x}{1-e^{-i x}} .
$$

An elementary calculation shows that

$$
h(x)=1+\frac{i x}{2}-\frac{x^{2}}{12}+O\left(x^{3}\right) .
$$

By using these explicit formulae, it is elementary to prove that there is a constant $C_{j} \approx 10$ so that the following estimates hold:

$$
\left|\tilde{g}_{2 M, k}-\hat{g}(k)\right| \leq \frac{C_{j}(|\alpha|+|\beta|)}{2 M+1} \text { for }|k| \leq M .
$$

Combining these estimates with those in Corollary 3.2, we obtain the following:

THEOREM 4.1 If $f$ is a piecewise continuous function on $[0,1]$ with jump part given by equation (4.1), then

$$
\left|\tilde{f}_{2 M, k}-\hat{f}(k)\right| \leq 6 \omega_{f^{c}}\left(\frac{1}{2 \pi M}\right)+\frac{C_{j} \sum_{j=1}^{p}\left(\left|\alpha_{j}\right|+\left|\beta_{j}\right|\right)}{2 M+1} \quad \text { for }|k| \leq M .
$$

If $f^{\mathrm{c}}$ is a Lipschitz-continuous, 1-periodic function, then

$$
\tilde{f}_{2 M, k}^{\mathrm{c}}=o\left(|k|^{-1}\right)
$$

uniformly in $M$, whereas

$$
\tilde{f}_{2 M, k}^{j}=\frac{\gamma_{M, k}}{k}+O\left(|k|^{-2}\right) .
$$

The absolute values of the coefficients $\left\{\gamma_{M, k}\right\}$ are generally bounded from below along sequences $\left(M_{j}, k_{j}\right)$ tending to $(\infty, \infty)$. This shows that the finite Fourier coefficients of a piecewise smooth function with jump discontinuities display the characteristic $\frac{1}{k}$-rate of decay.

If the function has a jump discontinuity, then the difference $\left|\tilde{f}_{2 M, k}-\hat{f}(k)\right|$ is, in fact, comparable to $\left|\tilde{f}_{2 M, k}\right|$ (or $\left.|\hat{f}(k)|\right)$ for $|k|$ close to $M$. With uniformly sampled data it is not possible to do better. Suppose that the sample spacing is $(2 M+1)^{-1}$ and $f(0)=0$ but $f\left((2 M+1)^{-1}\right)=1$. From this data, a jump could occur at any point in the interval $\left[0,(2 M+1)^{-1}\right]$. The difference $\left|\tilde{f}_{2 M, k}-\hat{f}(k)\right|$ can therefore be as large as

$$
\begin{aligned}
\Delta \tilde{f}_{k}=\left|\int_{0}^{\frac{1}{2 M+1}} e^{-2 \pi i k x}\right| & =\left|\frac{e^{-\frac{2 \pi i k}{2 M+1}}-1}{2 \pi i k}\right| \\
& \approx \frac{1}{2 M+1} \quad \text { for }|k| \leq M .
\end{aligned}
$$

The same line of reasoning shows that one cannot use the finite Fourier coefficients $\left\{\tilde{f}_{2 M, k}\right\}$ to locate the jumps in $f$ to an accuracy better than $(2 M+1)^{-1}$. On the other hand, in the work of Gottlieb et al., it is shown that, if $f$ is piecewise real analytic, 
then an exact knowledge of the Fourier coefficients $\{\hat{f}(-M), \ldots, \hat{f}(M)\}$ allows a reconstruction of $f$, away from its jump locus, with an exponentially small error, i.e., $O\left(e^{-a M}\right)$.

In the case of a function that is piecewise finitely differentiable with jump discontinuities, there are more sophisticated ways to use the sampled data and obtain more accurate approximations to the Fourier coefficients. Several such methods are described in [6, sec. 13.9] and [8, sec. 2.3.4]. Without going into excessive detail, these methods used the sampled data to approximate the function as a sum of higher-order interpolants. To approximate the Fourier coefficients, one need only compute the Fourier coefficients of the basic kernel functions used in the interpolation scheme. The approximation to $\hat{f}(k)$ is then expressed as a coefficient, depending on $M$ and $k$, times $\tilde{f}_{2 M, k}$ plus a finite sum of endpoint correction terms. These methods again presuppose an exact knowledge of the locations and sizes of the jumps.

As before, a uniform Riemann-Lebesgue lemma follows from these estimates.

COROLLARY 4.2 If $f$ is a piecewise continuous function on $[0,1]$, then there is a decreasing sequence $\left\langle a_{k}\right\rangle$ tending to 0 so that

$$
\left|\tilde{f}_{2 M, k}\right| \leq a_{k} \quad \text { for }|k| \leq M
$$

As a final result, we prove an analogue of the localization principle for the FFT partial sums.

THEOREM 4.3 (Localization Principle) Suppose that $f$ and $g$ are piecewise continuous functions defined on $[0,1]$, and suppose that $\tilde{g}_{2 M}(x)$ converges to $g(x)$. If

$$
y \mapsto \frac{f(y)-g(y)}{x-y}
$$

is continuous at $y=x$, then $\tilde{f}_{2 M}(x)$ converges to $f(x)$.

PROOF: The proof is very much like the continuous case. Because $f(x)=$ $g(x)$, it suffices to show that

$$
\lim _{M \rightarrow \infty}\left(\tilde{f}_{2 M}(x)-\tilde{g}_{2 M}(x)\right)=0 .
$$

Using formula (3.15) we obtain that

$$
\begin{aligned}
& \tilde{f}_{2 M}(x)-\tilde{g}_{2 M}(x) \\
& =\frac{1}{2 M+1} \sum_{j=0}^{2 M} D_{M}\left(x-\frac{j}{2 M+1}\right)\left[f\left(\frac{j}{2 M+1}\right)-g\left(\frac{j}{2 M+1}\right)\right] \\
& =\frac{1}{2 M+1} \sum_{j=0}^{2 M} \sin \left[(2 M+1) \pi\left(x-\frac{j}{2 M+1}\right)\right] \frac{\left[f\left(\frac{j}{2 M+1}\right)-g\left(\frac{j}{2 M+1}\right)\right]}{\sin \pi\left(x-\frac{j}{2 M+1}\right)}
\end{aligned}
$$




$$
=\Im\left[\frac{e^{(2 M+1) \pi i x}}{2 M+1} \sum_{j=0}^{2 M} e^{-\frac{2 \pi i M j}{2 M+1}}\left[\frac{e^{-\frac{\pi i j}{2 M+1}}\left[f\left(\frac{j}{2 M+1}\right)-g\left(\frac{j}{2 M+1}\right)\right]}{\sin \pi\left(x-\frac{j}{2 M+1}\right)}\right]\right] .
$$

The function defined by

$$
h(y)=\frac{e^{-\pi i y}[f(y)-g(y)]}{\sin \pi(x-y)}
$$

is piecewise continuous on $[0,1]$. The last line of equation (4.12) can be reexpressed as

$$
\tilde{f}_{2 M}(x)-\tilde{g}_{2 M}(x)=\Im\left[e^{(2 M+1) \pi i x} \tilde{h}_{2 M, M}\right],
$$

which, by virtue of Corollary 4.2 , tends to zero as $M$ tends to infinity. This proves the theorem.

\section{Nonuniformly Spaced Samples}

In this "experimental" section we show, via numerical experiments, that nonuniform sample spacing may lead to very different sorts of errors in the approximation of functions via Riemann sums for Fourier integrals. We consider the function $f$ with bounded support shown in Figure 5.1(a). Suppose that $\Xi=\left\{\xi_{j}: j=\right.$ $-J, \ldots, J\}$ is an increasing list of frequencies lying in the interval $\left[-\xi_{\max }, \xi_{\max }\right]$. We let

$$
|\Xi|=\max _{j}\left\{\xi_{j+1}-\xi_{j}\right\}
$$

denote the maximum sample spacing. Suppose that we can "measure" $\left\{\hat{f}\left(\xi_{j}\right)\right\}$; then the finite sum

$$
f_{\Xi}(t)=\frac{1}{2 \pi} \sum_{j=-J}^{J} \hat{f}\left(\xi_{j}\right) e^{i t \xi_{j}} w_{j}
$$

is a Riemann sum for the inverse Fourier transform applied to $\hat{f}$. Here $\left\{w_{j}\right\}$ are positive weights reflecting the sample spacing. If $|\Xi|$ is small enough to avoid aliasing, then $f_{\Xi}$ provides an approximation to $f$ over a sufficiently small interval. It seems quite interesting to understand how the properties of $\Xi$ affect the error $e_{\Xi}=f_{\Xi}-f$. For the present we consider several examples.

Our first examples are defined by choosing an even $\mathcal{C}^{1}$-function, $G(s)$, which is quadratic in an interval $\left[-s_{0}, s_{0}\right]$, satisfies $G(0)=0$, and is linear outside this interval. We set

$$
\xi_{j}=\operatorname{sign}(j) G(|j|)
$$

One such function is shown in Figure 5.1(b). In these examples, the error, $e_{\Xi}$, is larger than for the partial sum of the Fourier series defined by the uniformly spaced samples, with spacing $|\Xi|$, lying in $\left[-\xi_{\max }, \xi_{\max }\right]$. 


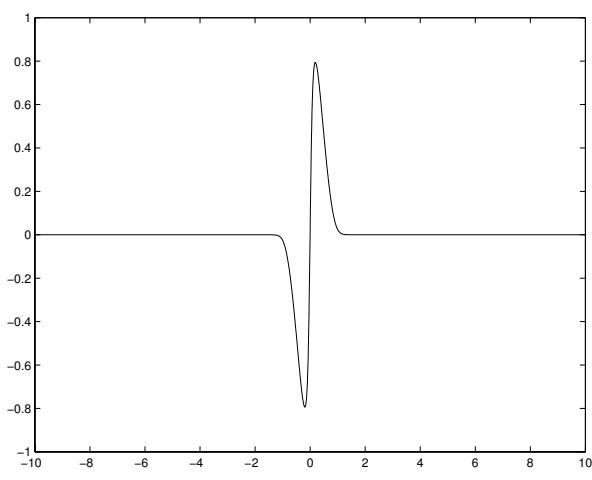

(a) Graph of the function $f(x)$.

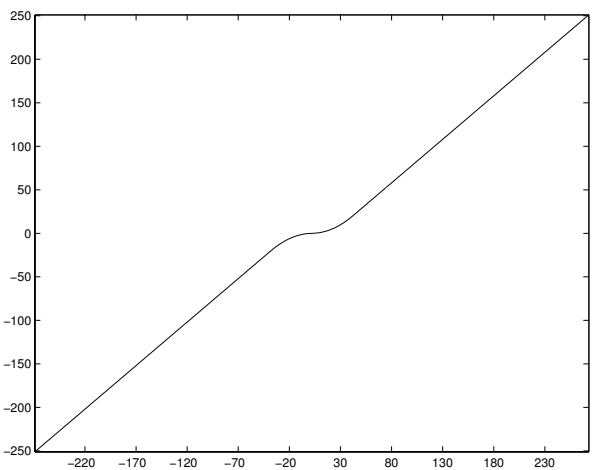

(b) An example of a frequency selection function, $G$.

FIGURE 5.1. Functions used in the construction of the examples.

Example 5.1. The function $f$ is a smoothed version of a function with a jump discontinuity, so, near to the jump in $f$, the error in the partial sum approximation is dominated by a Gibbs artifact; see Figure 5.2(a). Away from the jump, the error is proportional to the size of $f$. This is a reflection of the localization properties of the Fourier transform. If the transition from quadratic to linear growth occurs at a low frequency, then the error $e_{\Xi}$ is fairly evenly distributed. This is clearly shown in Figure 5.2(b). The maximum error in Figure 5.2(b) is about twice the maximum error in Figure 5.2(a). As the crossover frequency increases, the error more resembles the error in the partial sum; see Figure 5.2(c). Nonetheless, there remains a nonlocalized error that is considerably larger than for the partial sum. Note that the nonuniformly spaced sums have more terms than the uniformly spaced sums.

Example 5.2. For our second set of examples we slightly modify the definition of the frequencies. In the cases considered above, the uniform sample spacing, at high frequencies, is 1 . We modify the preceding examples by following the linearly spaced samples with one sample spaced by 1.1 , followed by one spaced by 0.9 , before going onto the uniformly spaced samples with spacing 1 . As shown in Figure 5.3, this slight irregularity in the sample spacing creates a much larger error, which grows rapidly as one moves away from the origin. The maximum error in Figure 5.3(a) is about 12 times that in Figure 5.2(b), and the maximum error in Figure 5.3(b) is about 4 times that in Figure 5.2(c). These examples indicate that minor changes in the sample spacing can have a profound effect on the quality of the approximation to a Fourier integral provided by a Riemann sum like that in $(5.1)$. 


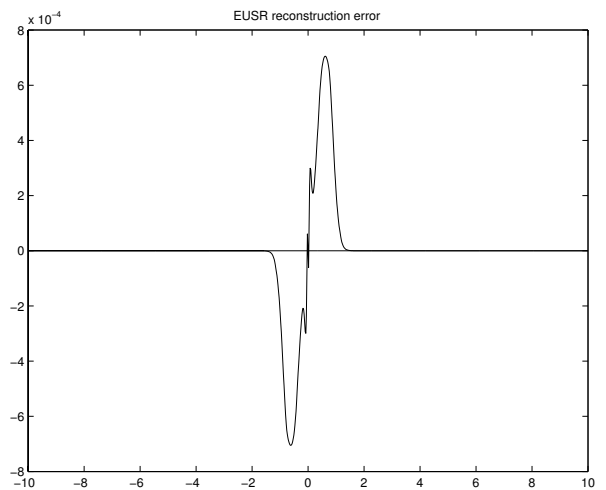

(a) The error in the partial sum with uniformly spaced samples, maxerr $\approx 7 \times$ $10^{-4}$.

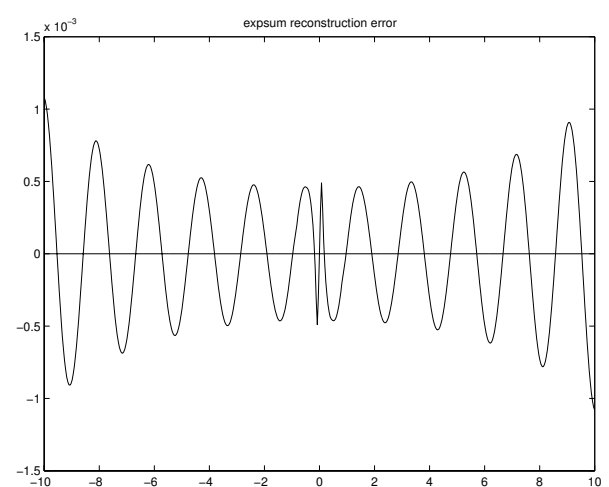

(b) The error with nonuniformly spaced samples, crossing over at 20 , maxerr $\approx$ $1.1 \times 10^{-3}$.

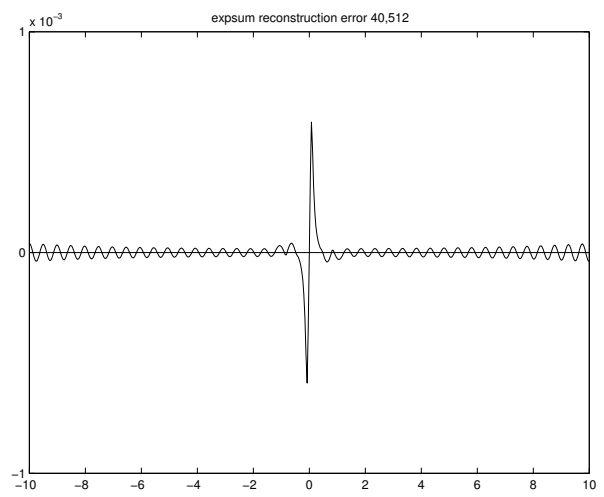

(c) The error with nonuniformly spaced samples, crossing over at 40 , maxerr $\approx$ $6 \times 10^{-4}$.

FIGURE 5.2. Comparison of the errors in the reconstructions obtained with uniformly and nonuniformly spaced samples. In these examples $\xi_{\max }=512$.

\section{Conclusion}

In this note we have established many basic properties of the finite Fourier transform in its role as an approximation to the Fourier transform. We have shown, in essence, that it behaves as well as it possibly could. The finite Fourier coefficients satisfy a uniform Riemann-Lebesgue lemma, and the FFT partial sums satisfy Riemann's celebrated localization principle. In examples we have seen that 


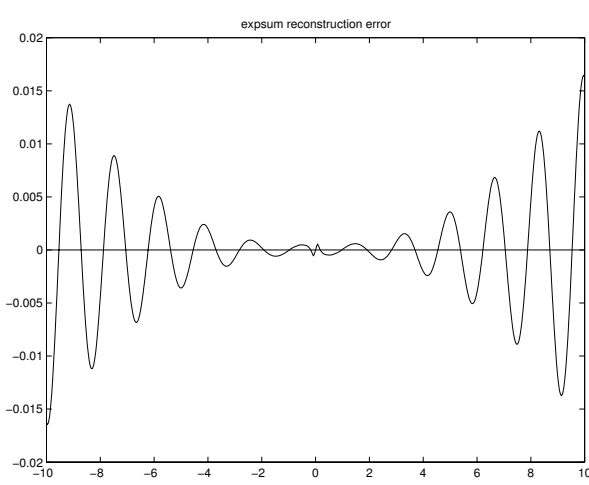

(a) The error with nonuniformly spaced samples, crossing over at 20, with two additional samples.

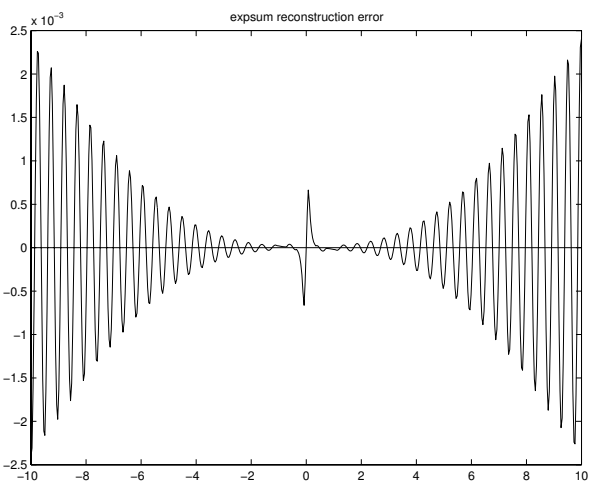

(b) The error with nonuniformly spaced samples, crossing over at 40 , with two additional samples.

FIGURE 5.3. Illustration of the effects on the error in approximation caused by a lack of monotonicity in the sample spacing.

nonuniformly spaced samples may lead to larger and qualitatively different errors. This seems a very interesting direction for further study. The trick used throughout the paper, which is embodied in Lemma 2.4, does not apply if the sample spacing is nonuniform.

Acknowledgment. I would like to thank Jeremy Magland for help with the numerical computations. I would also like to thank Arieh Iserles for the references to the work of Henrici, Gottlieb, and Shu, and Leslie Greengard, Yu Chen, and June-Yub Lee for several very helpful conversations on the finite Fourier transform. I would also like to thank the referee for several very useful and informative comments.

This research was partially supported by NSF Grant DMS02-03705.

\section{Bibliography}

[1] Auslander, L.; Grünbaum, F. A. The Fourier transform and the discrete Fourier transform. Inverse Problems 5 (1989), 149-164.

[2] Epstein, C. L. Introduction to the mathematics of medical imaging. Prentice Hall, Upper Saddle River, N.J., 2003.

[3] Gottlieb, D.; Shu, C-W. On the Gibbs phenomenon and its resolution. SIAM Review 39 (1997), 644-668.

[4] Henrici, P. Applied and computational complex analyis. Vol. 3. Wiley, New York, 1986.

[5] Katznelson, I. An introduction to harmonic analysis. Dover, New York, 1976.

[6] Press, W. H.; Teukolsky, S. A.; Vetterling, W. T.; Flannery, B. P. Numerical recipes in Fortran, the art of scientific computing. 2nd ed. Cambridge University, Cambridge, 1992.

[7] Rivlin, T. J. An introduction to the approximation of functions. Dover, New York, 1969. 
[8] Stoer, J.; Bulirsch, R. Introduction to numerical analysis. Springer, Heidelberg, 1980.

ChARles L. EPSTEIN

University of Pennsylvania

Department of Mathematics

209 South 33rd Street

Philadelphia, PA 19104-6395

E-mail: cle@math. upenn.edu

Received May 2004. 